\title{
Supersonic and Hypersonic Flight Dynamics Realization for the Spaceliner Real-Time Human-In-the-Loop Space Flight Simulator
}

\author{
Frank Morlang
}

\begin{abstract}
Objectives: DLR's real-time Human-in-the-Loop Space Flight Simulator needed an enhancement in its transonic and supersonic behavior for its advanced concept of a suborbital, hypersonic, winged passenger transport called SpaceLiner.

Methods/Statistical analysis: A simulation model has been developed by geometry modeled flight dynamics for the commercial flight simulation software "X-Plane". The presented solution is based on a real-time flight dynamics corrector application, taking table-based aerodynamic coefficients from Computational Fluid Dynamics (CFD) model experiments to overwrite X-Plane's internal flight dynamics in the supersonic and hypersonic regime.

Findings: Although compressible flow effects are considered using Prandtl-Glauert, the SpaceLiner X-Plane simulation model needed deeper investigation in its transonic and supersonic behavior, taking into account that transonic effects in X-Plane only refer to an empirical mach-divergent drag increase and the airfoil becomes an appropriate thickness ratio diamond shape under supersonic conditions. Whereas the X-Plane internal flight simulation engine delivers a high level of realism under subsonic conditions, significant deviations from the SpaceLiner aerodynamic reference database were identified in the supersonic and hypersonic regimes. An improved accuracy could be observed for two Mach test cases under corrector application usage conditions. Using X-Plane on the one hand and covering a constant accuracy throughout the whole range of regimes, subsonic, supersonic and hypersonic on the other hand, can be achieved by using the presented corrector application solution.

Application/Improvements: X-Plane's wireframe model approach was successfully fused with table-based lookup processing, delivering a constant high level of realism throughout the whole Mach range.
\end{abstract}

Keywords: flight dynamics, supersonic, hypersonic, real-time, flight simulator

\section{INTRODUCTION}

The SpaceLiner [1], DLR's advanced concept for a suborbital, hypersonic, fix-winged passenger plane was depicted for the use of deeper investigations referring the integration of future space traffic into normal air traffic. Especially with respect to emergency situations [2], the influences of future air/ground information sharing on human factors aspects must be regarded under real-time-human-in-the-loop flight simulation conditions. To address this need, a SpaceLiner geometric model (Figure 1) was developed with the help of the X-Plane flight simulator tool called Plane Maker.

Revised Version Manuscript Received on 16 September, 2019.

Frank Morlang, German Aerospace Center (DLR), E-Mail: frank.morlang@dlr.de

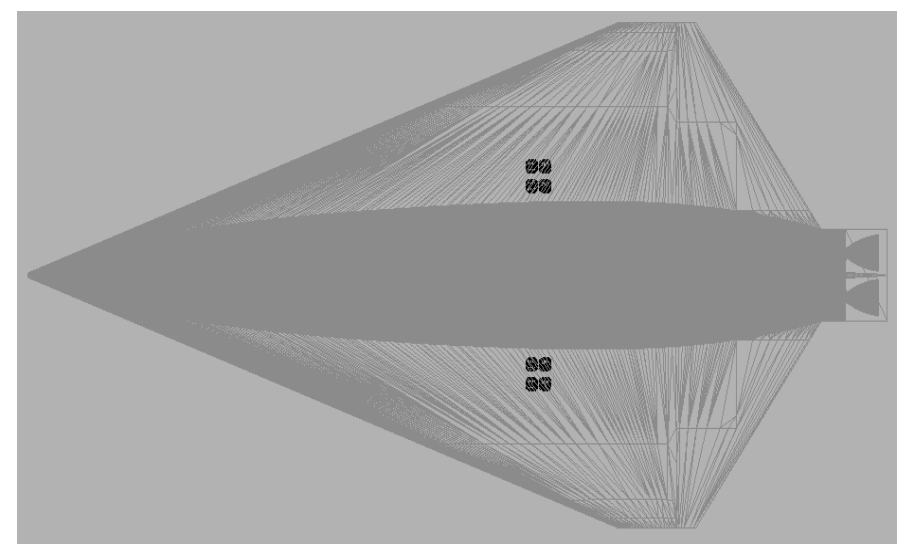

Figure 1. SpaceLiner model in X-Plane's Plane Maker tool

The X-Plane internal flight simulation engine processing reads in the geometric wireframe model and derives its flight dynamics model (FDM) by dividing the geometry in many small parts and summing up the acting forces on each part. Accelerations are derived; velocities are calculated by integration over time and double integration over time results in the positions. This technique delivers a high level of realism in the subsonic regime (Figure 2 and Figure 3 ).

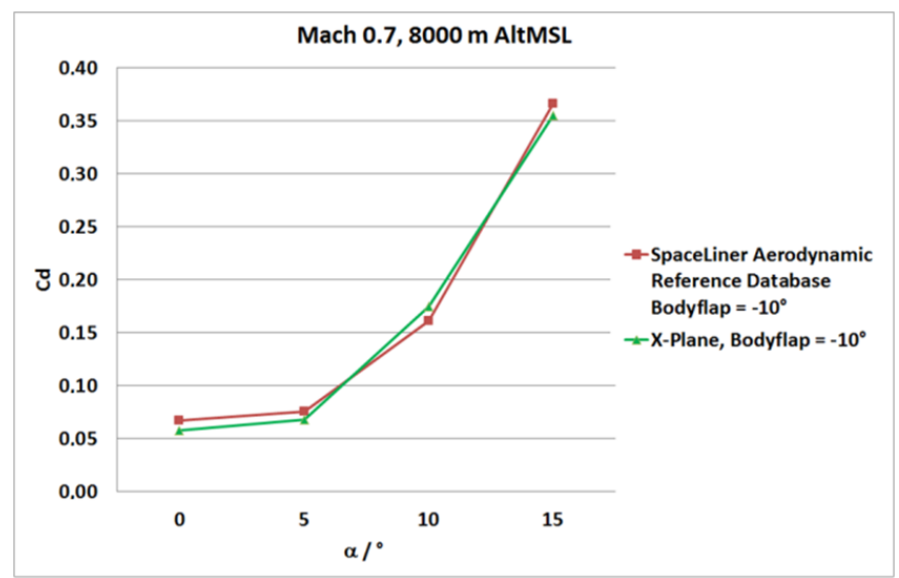

Figure 1. Coefficient of $\operatorname{drag}\left(\mathrm{C}_{\mathrm{d}}\right)$ vs. $\alpha$ SpaceLiner aerodynamic reference database / $\mathrm{X}$-Plane model comparison 


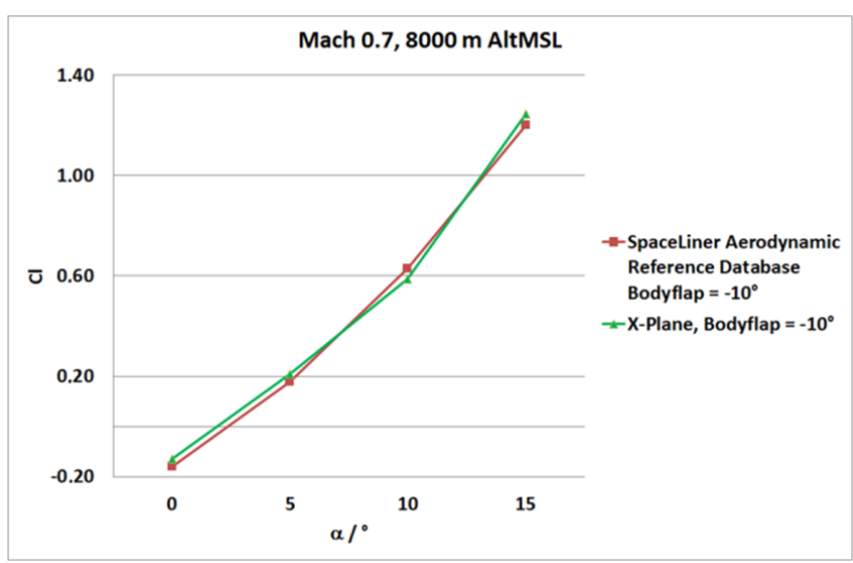

Figure 2. Coefficient of lift $\left(C_{1}\right)$ vs. $\alpha$ SpaceLiner aerodynamic reference database / $\mathrm{X}$-Plane model comparison

Although X-Plane considers compressible flow effects, this realistic accuracy is not realized in the supersonic and hypersonic regimes (Figure 4 and Figure 5), because only an appropriate thickness ratio diamond shape, representing the airfoil under supersonic conditions, is used to model an empirical Mach-divergent drag increase.

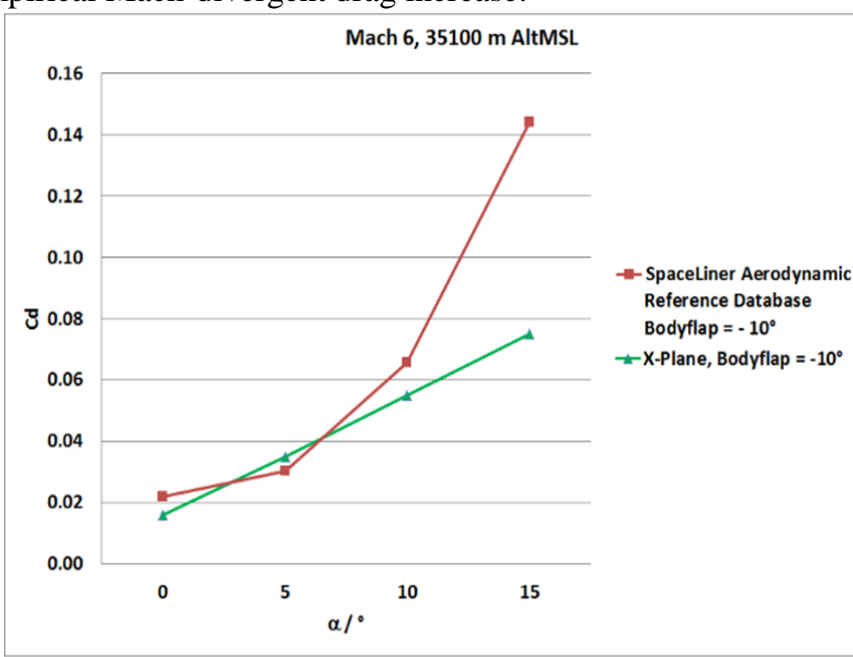

Figure 3. $C_{d}$ vs. $\alpha$ SpaceLiner aerodynamic reference database / X-Plane model comparison

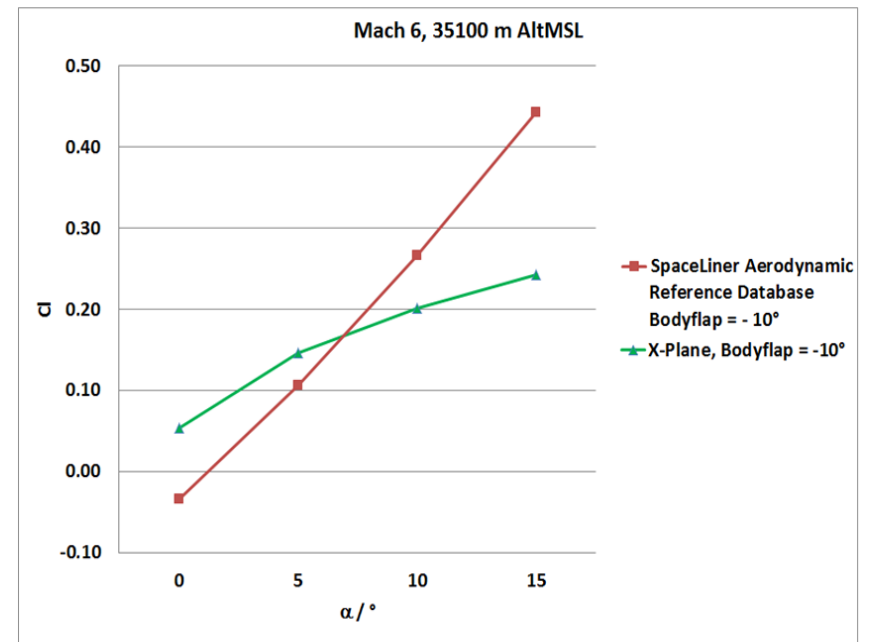

Figure 4. $C_{1}$ vs. $\alpha$ SpaceLiner aerodynamic reference database / X-Plane model comparison

\section{CHALLENGE}

The challenge is to cover a constant accuracy throughout the whole range of regimes, subsonic, supersonic and hypersonic. Additional lift and drag generating parts for deployment in different Reynolds number ranges can be modeled in X-Plane's Plane Maker tool, thus a flight dynamics model tuning for a best fit of the complete reference data set is possible this way. It must be pointed out that this approach is mainly characterized by trial-and-error, embedding the risk of a very cost- and time-consuming process. Against the mentioned background, model tuning by creating additional parts in Plane Maker was regarded as an out of scope process and therefore rejected.

\section{SOLUTION}

Facing the fact that the X-Plane environment has powerful User Datagram Protocol (UDP) input/output (i/o) capabilities, addressing data read in as well as overwriting data with external content [3], a CD, CL corrector application was realized. This solution reads the speed and angle of attack ( $\square$ ) of the X-Plane flight dynamics model engine and injects corrected CD and CL values with the associated SpaceLiner aerodynamic reference database [4] data (Figure 6), derived from Computational Fluid Dynamics (CFD), Calculation of Aerodynamic Coefficients (CAC) \& PAN AIR model experiments' results.

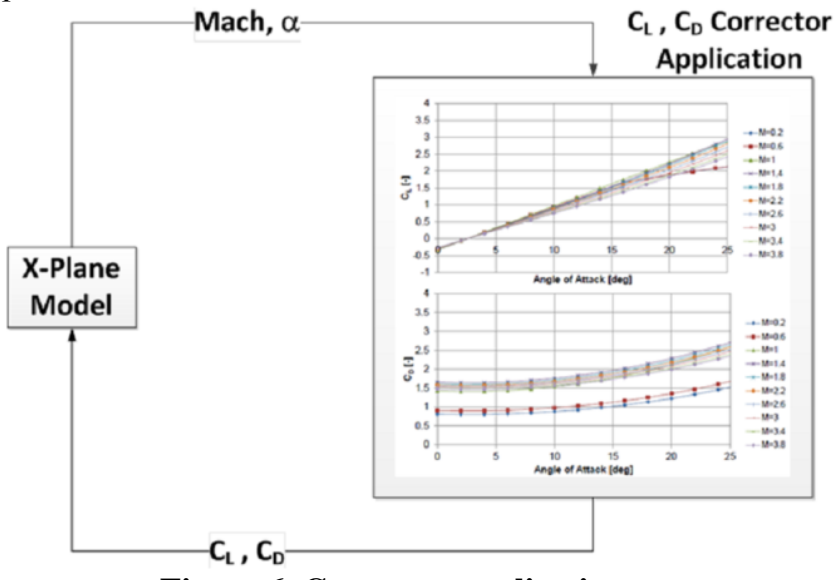

Figure 6. Corrector application

The $C_{L}, C_{D}$ corrector application was developed in $\mathrm{Tcl}$ (Tool command language), using Tcllib's math::interpolate package for finding the Mach and $\alpha$ dependent coefficient data Error! Reference source not found.. The used aerodynamic reference data range for interpolation is shown in Error! Reference source not found.

Table 1. Tabled CL, CD data

\begin{tabular}{|c|c|c|c|c|c|c|c|c|c|}
\hline \multirow[b]{2}{*}{$\alpha$} & \multicolumn{9}{|c|}{ Mach } \\
\hline & $\begin{array}{l}1 . \\
1\end{array}$ & $\begin{array}{l}2 . \\
0\end{array}$ & $\begin{array}{l}4 . \\
0\end{array}$ & $\begin{array}{l}6 . \\
0\end{array}$ & $\begin{array}{l}10 . \\
0\end{array}$ & $\begin{array}{l}14 . \\
0\end{array}$ & $\begin{array}{l}18 . \\
0\end{array}$ & $\begin{array}{l}22 \\
0\end{array}$ & $\begin{array}{l}26 . \\
0\end{array}$ \\
\hline 0.0 & $X$ & $\cdots$ & $\cdots$ & $\cdots$ & $\cdots$ & $\cdots$ & $\cdots$ & $\ldots$ & $\cdots$ \\
\hline 5.0 & $\ldots$ & $\ldots$ & $\ldots$ & $\ldots$ & $\ldots$ & $\ldots$ & $\ldots$ & $\ldots$ & $\ldots$ \\
\hline 10.0 & $\ldots$ & $\ldots$ & $\ldots$ & $\ldots$ & $\ldots$ & $\ldots$ & $\ldots$ & $\ldots$ & $\ldots$ \\
\hline
\end{tabular}




\begin{tabular}{|l||l|l|l|l|l|l|l|l|l|}
\hline 15.0 & $\mathrm{X}$ & $\cdots$ & $\cdots$ & $\cdots$ & $\cdots$ & $\cdots$ & $\cdots$ & $\cdots$ & $\mathrm{X}_{4,9}$ \\
\hline 4,1 & & & & & & & & \\
\hline
\end{tabular}

\section{RESULTS AND DISCUSSION}

The solution's performance test refers to a local test set-up (Error! Reference source not found.) and covers the following processing actions:

- $\quad$ Read out Mach and angle of attack ( $\square$ ) of the $\mathrm{X}$-Plane generated flight dynamics model

- Table based $C_{L}, C_{D}$ coefficient interpolation

Overwriting X-Plane $C_{L}, C_{D}$ values with the found interpolation dataAnalyzing the results (Figure 7) in combination with the resolving X-Plane's transport delay of peaks not higher than $30 \mathrm{~ms}$ (Figure 8) reveals the fulfilling of the flight simulation training device request that the transport delay must not exceed $150 \mathrm{~ms}$ [6].

Table 2. Test set-up parameters

\begin{tabular}{|l|l|}
\hline Computer & $\begin{array}{l}\text { Dell Latitude E6430 } \\
\text { 8192 MB memory } \\
\text { Intel® CoreTM i7-3520M CPU @ 2.90 } \\
\text { GHz } \\
\text { Windows 7 Enterprise OS (SP 1) }\end{array}$ \\
\hline $\begin{array}{l}\text { X-Plane } \\
\text { version }\end{array}$ & $\begin{array}{l}\text { 10.51, 64 bit with medium rendering } \\
\text { options set (NVIDIA NVS 5200M } \\
\text { graphic card) }\end{array}$ \\
\hline $\begin{array}{l}\text { Tcl/Tk } \\
\text { version }\end{array}$ & Active Tcl 8.6.9 Build 8609.2 (64 bit) \\
\hline
\end{tabular}

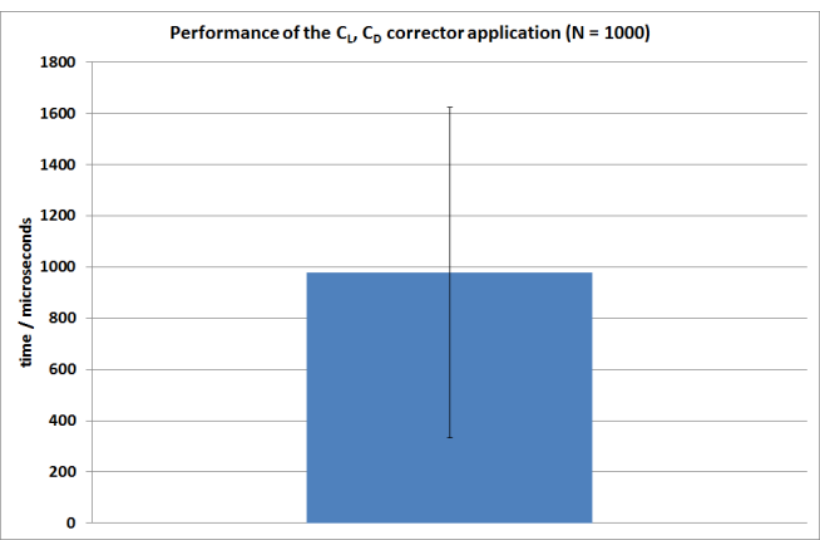

Figure 7. Performance of the $C_{L}, C_{D}$ corrector application

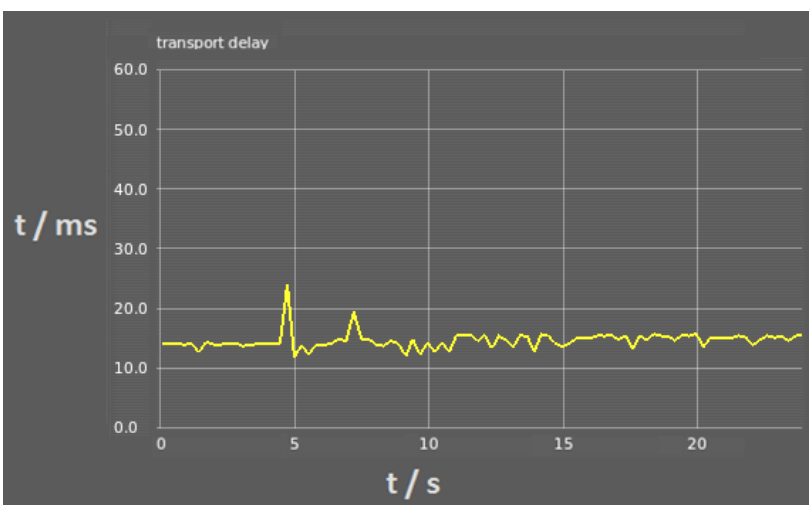

Figure 8. Transport delay vs. time

The coefficient results for the two test cases Mach 6 and 18 are presented in Figure 9 to Figure 12, showing an improved accuracy throughout the $\alpha$ corridor, ranging from 0 to 15 degrees. Because of X-Plane internal additional noise on top of overwritten data, the coefficients for the tabled $\alpha$ values of $0,5,10$ and 15 degrees are not exactly met.

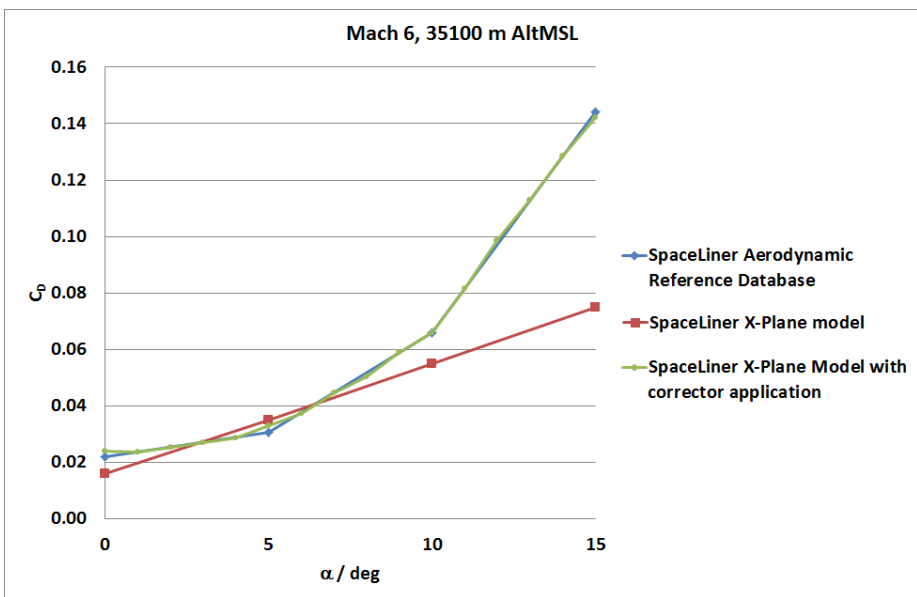

Figure 9. Mach $6 C_{D}$ comparison

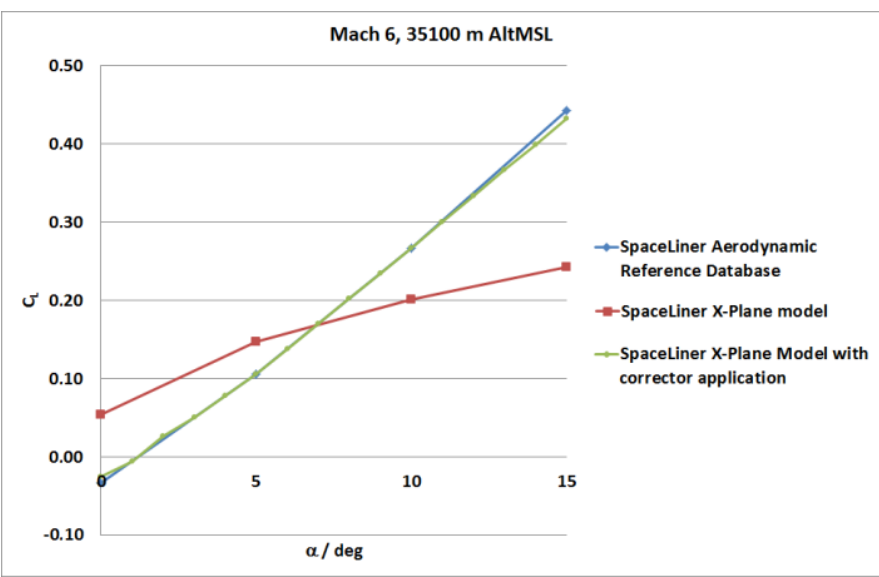

Figure 10. Mach $6 \mathrm{C}_{\mathrm{L}}$ comparison

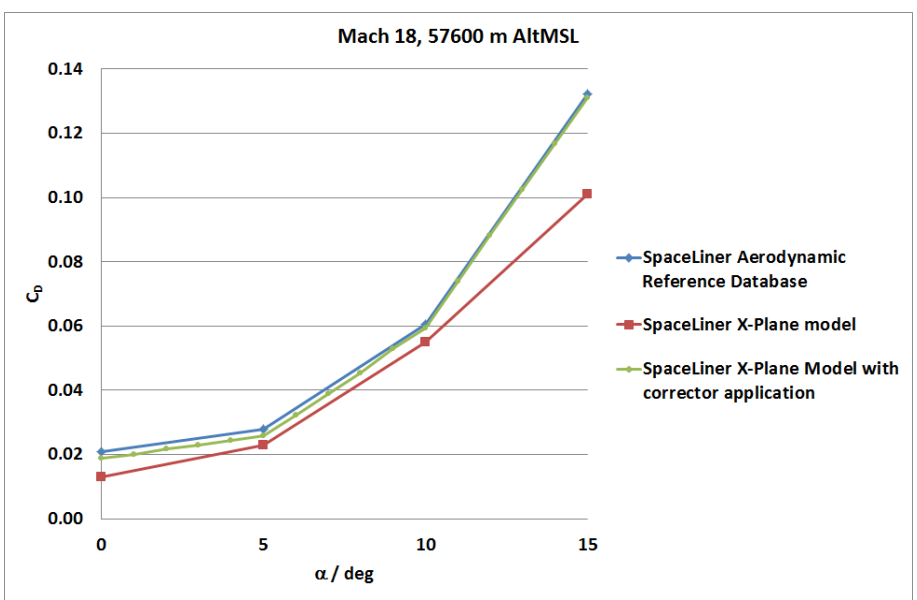

Figure 11. Mach $18 \mathrm{C}_{\mathrm{D}}$ comparison

Published By: 


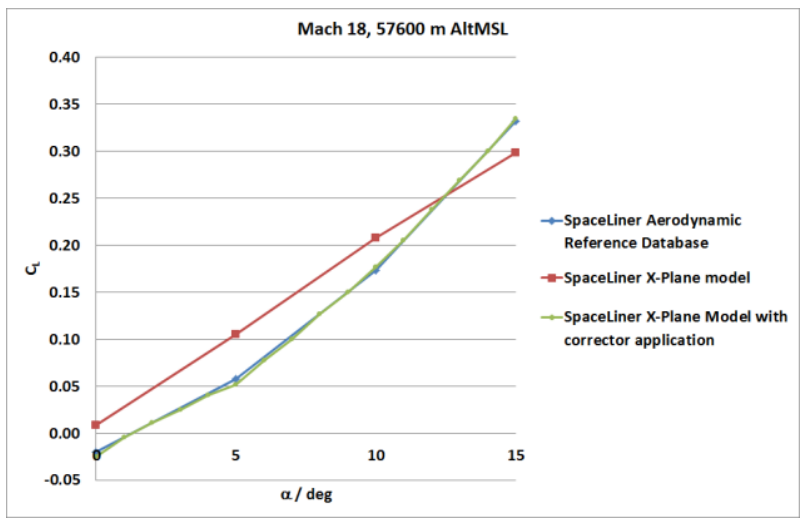

Figure 12. Mach $18 \mathrm{C}_{\mathrm{L}}$ comparison

Supersonic and hypersonic flight dynamics for the SpaceLiner real-time Human-in-the-Loop simulator were successfully realized, opening the possibility to combine X-Plane's wireframe model approach with table-based lookup processing. The strengths of both techniques in their associated dynamic regimes were successfully fused, performing a constant high level of realism throughout the whole $\alpha$ - Mach range.

\section{CONCLUSION}

The actual $\alpha$ and Mach relations in the SpaceLiner aerodynamic reference database are based on a $\beta$ value of 0 degrees. The corrector application is already prepared to read in data sets covering $\alpha$ - Mach tables for a set of $\beta$ ranges, thus future aerodynamic reference database updates will directly result in related corrector application ones.

\section{REFERENCES}

1. M. Sippel, J. Klevanski. and J. Steelant, Editors. Comparative Study on Options for High-Speed Intercontinental Passenger Transports: Air-Breathingvs. Rocket-Propelled. Proceedings of 56th International Astronautical Congress, (2005); Fuduoka, Japan

2. F. Morlang, J. Ferrand and R. Seker, The Journal of Space Safety Engineering 4, 5-8 (2016)

3. X-Plane 11 Desktop Manual "Data Input and Output from X-Plane",11th of June 2019. [Online]. Available: https://www.x-plane.com/manuals/desktop/\#expandin gx-plane.html. [Accessed 15 Aug. 2019].

4. T. Schwanekamp, L. Morsa, G. Zuppardi and R. Molina, Editors. SpaceLiner 7-2 Aerodynamic Reference Database. Proceedings of SART TN-026/2012, (2012); Bremen, Germany

5. F. Morlang, Editor. Quick and dirty I/O playing with X-Plane without "dirty" - the Tcl way. Proceedings of 26th Tcl Conference, (2019) November 05-08; Houston, USA

6. European Aviation Safety Agency, Editor, Certification Specifications for Aeroplane Flight Simulation Training Devices, Annex to ED Decision 2012/010/, (2012)

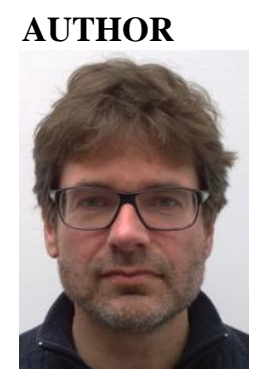

Frank Morlang

1999 Diploma in Engineering in Materials Science of Technical University of Darmstadt 1999 - 2001 Project engineer at Aerodata company Braunschweig 2001 - today Researcher at Deutsches Zentrum für Luft- und Raumfahrt (German Aerospace Center), DLR, Braunschweig 